\title{
Comparação da composição, dos aspectos nutricionais e do preço de mercado entre o
} leite UHT e bebidas vegetais UHT

\author{
Comparison of composition, nutritional aspects and market price between UHT milk and UHT \\ plant-based beverages \\ Comparación de composición, aspectos nutricionales y precio de mercado entre leche UHT y \\ bebidas vegetales UHT
}

Recebido: 21/09/2021 | Revisado: 01/10/2021 | Aceito: 05/10/2021 | Publicado: 07/10/2021

Luiza Oliveira Mello

ORCID: https://orcid.org/0000-0001-8629-3681

Universidade Federal de Juiz de Fora, Brasil E-mail: oliveiramelloluiza@gmail.com Júlia d'Almeida Francisquini

ORCID: https://orcid.org/0000-0001-6680-945X

Universidade Federal de Juiz de Fora, Brasil

E-mail: juliafrancisquininutri@gmail.com

Juliana de Carvalho da Costa

ORCID: https://orcid.org/0000-0002-2336-7361

Universidade Federal de Juiz de Fora, Brasil

E-mail: juliana.costa@ufjf.br

Daniela Kharfan

ORCID: https://orcid.org/0000-0001-9104-9368 John Bean Technologies Máquinas e Equipamentos Industriais Ltda, Brasil E-mail: daniela.kharfan@jbtc.com

Ítalo Tuler Perrone

ORCID: https://orcid.org/0000-0002-3393-4876

Universidade Federal de Juiz de Fora, Brasil E-mail: italotulerperrone@gmail.com

Rodrigo Stephani

ORCID: https://orcid.org/0000-0003-0237-8325

Universidade Federal de Juiz de Fora, Brasil E-mail: rodrigostephani@gmail.com

\begin{abstract}
Resumo
Cresce a demanda por substitutos do leite por pessoas que alegam preocupações ambientais, sustentáveis ou que exibem algum tipo de restrição alimentar como veganos, vegetarianos, intolerantes à lactose ou alérgicos à proteína do leite. Dentro destes substitutos aumenta-se o consumo de bebidas vegetais as quais ainda carecem de uma legislação específica e consolidada para controle de sua identidade e qualidade o que já é estabelecido para o leite UHT. Sendo assim, o objetivo do trabalho foi realizar um estudo comparativo das informações disponibilizadas nos rótulos de bebidas vegetais UHT escolhidas (cinco tipos de fontes vegetais produzidas por três diferentes empresas) e de leites de vaca UHT (três tipos de leite produzidos por cinco diferentes empresas) no que tange os aspectos nutricionais, tempo de validade total, claims, lista de ingredientes, denominação de venda e preço de mercado. Foram realizadas análises estatísticas e exploratórias dos dados obtidos. Pôde-se verificar que as bebidas vegetais apresentaram preço médio por litro de produto aproximadamente 3,4 vezes mais elevado que os leites UHT, e que as mesmas, exibiram maior quantidade de ingredientes adicionados e variações em sua composição nutricional demonstrando a necessidade de uma legislação específica. Por meio da análise de componentes principais, as amostras de bebidas vegetais de soja se agruparam às amostras de leite UHT por possuírem atributos de composição mais próximo deste produto de origem animal. Portanto, verifica-se um nicho a ser explorado na busca de uma maior padronização e diminuição do preço de mercado das bebidas vegetais.
\end{abstract}

Palavras-chave: Rótulo; Legislação; Substitutos do leite.

\begin{abstract}
The demand for milk substitutes is growing by people who claim environmental concerns, sustainability or who exhibit some type of food restriction such as vegans, vegetarians, lactose intolerant or allergic to milk protein. Within these substitutes, the consumption of vegetable drinks is increased, which still lack specific and consolidated legislation to control their identity and quality, which is already established for UHT milk. Thus, the objective of the
\end{abstract}


work was to carry out a comparative study of the information available on the labels of vegetable beverages (five types of vegetable sources produced by three different companies) and UHT cow's milk (three types of milk produced by five different companies) regarding nutritional aspects, total shelf life, claims, ingredients list, sales denomination and market price. Statistical and exploratory analyzes of the data obtained were performed. It could be verified that vegetable drinks have an average price per liter of product approximately 3.4 times higher than UHT milks, and that they exhibited a greater amount of added ingredients and variations in their nutritional composition, demonstrating the need for specific legislation. Through principal component analysis, the samples of soy vegetable drinks were grouped with the UHT milk samples for having compositional attributes closer to that of animal-derived product. Therefore, there is a niche to be explored in the search for greater standardization and a reduction in the market price of vegetable drinks.

Keywords: Label; Legislation; Milk substitutes.

\section{Resumen}

La demanda de sucedáneos de la leche está creciendo por parte de personas que reclaman preocupaciones ambientales, sustentabilidad o que exhiben algún tipo de restricción alimentaria como veganos, vegetarianos, intolerantes a la lactosa o alérgicos a la proteína de la leche. Dentro de estos sustitutos se incrementa el consumo de bebidas vegetales, que aún carecen de una legislación específica y consolidada para controlar su identidad y calidad, lo que ya es establecido para la leche UHT. Así, el objetivo del trabajo fue realizar un estudio comparativo de la información disponible en las etiquetas de bebidas vegetales (cinco tipos de fuentes vegetales producidas por tres empresas diferentes) y leche de vaca UHT (tres tipos de leche producidos por cinco empresas diferentes) en cuanto a aspectos nutricionales, vida útil total, declaraciones, lista de ingredientes, denominación de venta y precio de mercado. Se realizaron análisis estadísticos y exploratorios de los datos obtenidos. Se pudo verificar que las bebidas vegetales tienen un precio promedio por litro de producto aproximadamente 3,4 veces mayor que las leches UHT, y que exhiben una mayor cantidad de ingredientes agregados y variaciones en su composición nutricional, demostrando la necesidad de una legislación específica. A través del análisis de componentes principales, las muestras de bebidas vegetales de soja se agruparon con las muestras de leche UHT por tener atributos de composición más cercanos de este producto de origen animal. Por tanto, hay un nicho a explorar en la búsqueda de una mayor estandarización y reducción del precio de mercado de las bebidas vegetales.

Palabras clave: Etiqueta; Legislación; Sucedáneos de la leche.

\section{Introdução}

Atualmente cresce por parte dos consumidores a busca por empresas que alegam alguma preocupação ambiental e sustentável na sua sequência de produção, desde a chegada da matéria prima até venda do produto final (Mcclements, Newman \& Mcclements, 2019). Em associação, a procura por alimentos mais saudáveis e de rápido consumo, propiciam o desenvolvimento das indústrias de alimentos, que visam contribuir com os objetivos de seus consumidores: alcance de uma dieta prática e com qualidade nutricional (Casé, et al., 2005). A sociedade tem alterado seu padrão de alimentação buscando associação entre praticidade com consumo de alimentos saudáveis e produzidos por empresas com responsabilidade ambiental.

Acompanhando estas tendências, observa-se um aumento na quantidade de veganos, vegetarianos, intolerantes à lactose e/ou alérgicos à proteína do leite de vaca, ou seja, pessoas que almejam alternativas na ingestão do leite e seus derivados (Paul, et al., 2019). Ao analisar as opções de substitutos do leite para estes indivíduos, destacam-se as bebidas à base de vegetais. Conforme divulgado pelo site especializado Milkpoint (2018) houve um crescimento de 51,5\% do mercado de bebidas vegetais no país em 2018, com destaque para bebidas produzidas a partir de arroz, aveia, coco e amêndoas. Tais bebidas, de forma geral, possuem um preço muito elevado ao ser comparado ao preço do leite de vaca (Munekata, et al., 2020). Portanto, as realidades expostas, refletem no consumo de leite ultra alta temperatura (UHT - Ultra High Temperature), assim como no das bebidas vegetais, impactando no processo de produção e compra desses alimentos.

As bebidas vegetais não podem ser consideradas um substituto direto dos leites UHT, no que diz respeito ao valor nutricional e preço de mercado (Cirilo, et al., 2020). Contudo, algumas bebidas vegetais obtidas a partir de leguminosas, como a soja, podem proporcionar teor proteico semelhante ao leite de vaca. Além disto, quando tais bebidas são enriquecidas com cálcio, vitamina D e vitamina B12, tendem a ser consideradas substitutas equivalentes dos leites UHT. Sendo assim, verifica-se um nicho a ser explorado por parte das indústrias de alimentos na busca por novos produtos, na tentativa de elaboração e 
diversificação nas bebidas vegetais com valor nutricional agregado e mais acessível do ponto de vista financeiro (Munekata, et al., 2020).

Vale ressaltar que já existem padrões de identidade, qualidade e rotulagem definidos para leites de vaca UHT pela Portaria n 370/1997 (Brasil, 1997). Em contrapartida, até o momento não há legislações específicas para a padronização das bebidas vegetais, existindo apenas algumas normas voltadas à padronização de alguns produtos proteicos de origem vegetal, em especial soja e trigo ( $R D C n^{\circ}$ 268/2005) e descrição de requisitos específicos (pH e umidade), boas práticas de fabricação e rotulagem de produtos de vegetais, produtos de frutas e cogumelos comestíveis ( $R D C n^{\circ}$ 272/2005). Com relação às bebidas, a Lei $n^{\circ}$ 8.918/1994, dispõe sobre a padronização, a classificação, o registro, a inspeção, a produção e a fiscalização de bebidas em geral.

A partir do exposto, o objetivo do presente trabalho foi realizar um estudo comparativo das informações disponibilizadas nos rótulos de bebidas vegetais UHT e de leites de vaca UHT no que tange os aspectos nutricionais, tempo de validade total, claims, lista de ingredientes, denominação de venda e preço de mercado.

\section{Metodologia}

O trabalho baseia-se em um trabalho de campo por meio da análise de bebidas vegetais UHT de cinco diferentes fontes (amêndoa, arroz, castanha de caju, coco e soja) de três diferentes marcas para cada fonte vegetal (totalizando 15 produtos) foram adquiridas no mercado local da Zona da Mata do Estado de Minas Gerais assim como também via compra online com entrega na mesma região. Em relação aos leites UHT, foram analisados três tipos (integral, semidesnatado e desnatado) de cinco diferentes marcas para cada tipo de leite (totalizando 15 produtos), obtidos também em mercado local da Zona da Mata do Estado de Minas Gerais (Ludke \& Andre, 2013).

Foram analisadas as rotulagens de cada bebida vegetal e leite UHT, considerando a denominação de venda, alegações e atributos no display frontal, informações nutricionais (valor energético, carboidratos, proteínas, gorduras, gorduras saturadas, fibras, sódio e cálcio), lista de ingredientes, instrução de tempo de consumo após a abertura do produto e tempo de validade total (calculado em dias). Além das informações contidas no rótulo, também foram analisados os preços disponibilizados ao consumidor no momento da compra entre os meses de maio e julho de 2021.

Foi realizada análise estatística para determinação de média geral, valor mínimo e máximo, amplitude, desvio padrão, erro padrão da média e coeficiente de variação por meio do software R versão 4.1.1 (2020). Realizou-se também a análise exploratória por meio da análise de componentes principais (PCA) usando o software Matlab versão 7.10.0 (2010). Os dados (valor energético, teor de carboidratos, proteínas, gorduras, gorduras saturadas, fibras, sódio e cálcio) foram analisados sem a necessidade de pré-processamento. No modelo construído pelo PCA, foram escolhidos dois componentes principais PC 1 $(95,53 \%)$ e PC 2 (3,49\%). A escolha dos PCs foi baseada no gráfico de autovalores versus número de componentes principais.

\section{Resultados e Discussão}

$\mathrm{Na}$ Tabela 1, são apresentados os dados gerais sobre as quinze amostras de bebidas à base de vegetais e quinze leites UHT, exibindo os códigos das amostras, denominação de venda, preço de venda, tempo de validade total (em dias) e tempo de consumo após aberto (em dias) indicada pelo fabricante. Nota-se que as bebidas vegetais apresentaram preço médio por litro de produto ( $\mathrm{R} \$ 14,32)$ aproximadamente 3,4 vezes mais elevado que os leites UHT (média de preço R 4,18 ). Dentre as bebidas vegetais, a que exibiu o valor mais elevado ( $\mathrm{R} \$ 26,56)$ foi a de castanha de caju ( $\mathrm{C} 1$ - alimento com castanha de caju orgânico), enquanto o valor mais baixo encontrado foi de $\mathrm{R}$ \$,99 para bebidas formuladas à base de amêndoa (A1 - alimento com amêndoa) e soja (E1 - alimento com soja). Em relação aos leites UHT o preço mais alto encontrado foi de R\$ 6,79 e o 
mais baixo de $\mathrm{R} \$ 2,99$, ambos para produtos semidesnatados.

Para obtenção das bebidas vegetais existem diferentes etapas de processamento (moagem úmida, filtração, adição de ingredientes, tratamento térmico, homogeneização, envase asséptico, armazenamento refrigerado, descasque, torrefação, moagem a seco, maceração em ácido diluído, adição de enzimas, imersão em água deionizada, entre outros) com aplicação de distintas tecnologias incluindo UHT, ultrassom, campo elétrico pulsado, aquecimento ôhmico, homogeneização de alta e ultra alta pressão. Em associação, desafios como o alto valor comercial agregado aos vegetais usados e a dificuldade de se realizar fabricações em grande escala, são fatores associados ao encarecimento destas bebidas vegetais (Aydar, et al., 2020).

Também é possível observar na Tabela 1 que o tempo de validade, representado em dias, foi cerca de 2,4 vezes maior para as bebidas vegetais (312 dias em média) do que para os leites UHT (131 dias em média). Destaca-se que os leites exibiram em todas as amostras a data de fabricação e validade, mas em contrapartida, $60 \%$ das bebidas vegetais não apresentaram as datas de fabricação (apenas validade final), uma vez que a mesma não é de caráter obrigatório nos rótulos de alimentos embalados (RDC n 259/2002).

No que diz respeito ao tempo de consumo dos produtos após sua abertura, para todos os leites UHT, o valor informado foi de dois dias, enquanto para as bebidas vegetais o tempo de consumo após aberto variou entre dois e sete dias, sendo que duas amostras não especificaram o tempo de geladeira (B3 e C3) e uma amostra não informou como conservar o produto após aberto (A3). Isto já é um primeiro indicativo da falta de padronização de identidade, qualidade e rotulagem das amostras de bebidas vegetais pela ausência de uma regulamentação brasileira específica para estes produtos.

Na Tabela 2, estão demonstrados os valores nutricionais das bebidas vegetais, padronizados para uma porção de 100 $\mathrm{ml}$, a partir das informações disponibilizadas nos rótulos dos produtos. Verifica-se que as bebidas à base de vegetais exibiram variações em sua composição até mesmo quando foi utilizada uma mesma fonte vegetal (coeficientes de variação elevados não apresentados na tabela). Isto reafirma que as indústrias produtoras destas bebidas carecem de uma legislação específica para evitar que as mesmas tenham valores energéticos e nutricionais variáveis entre si, independentemente de serem formuladas com diferentes ou iguais fontes vegetais.

Dentre as bebidas vegetais, a de soja (E3) apresentou os maiores valores proteicos (valor médio de $2,7 \mathrm{~g} / 100 \mathrm{ml}$ ). O nível de gordura foi maior na bebida $\mathrm{C} 1$ que possui como fonte vegetal a castanha de caju (valor médio de 3,7 g/100 ml). Além disto, o maior valor de carboidratos pertence a bebida vegetal B1 obtida à base de arroz e a maior quantidade de fibras é identificada na bebida D1 (produzida a base da fonte vegetal coco). Os maiores valores de sódio e de cálcio, respectivamente, estão nas bebidas vegetais à base de soja (E1) e amêndoa (A2).

Avaliando os coeficientes de variação dentro de uma mesma fonte vegetal, infere-se que a menor variação foi encontrada na bebida a base de arroz para o teor de carboidrato (18\%), na bebida a base de soja para o teor de proteína (18\%), lipídeo $(10 \%)$, sódio $(21 \%)$, cálcio $(6 \%)$ e na bebida a base de amêndoa para o teor de fibra (0\%). Portanto, uma maior uniformidade no que diz respeito aos atributos nutricionais pode ser encontrada nas bebidas vegetais formuladas por meio da soja.

Alimentos à base de vegetais exibem potenciais aplicações à saúde por seus benefícios conferidos pelas fibras dietéticas. A fibra dietética é parte integrante e obrigatória de uma dieta saudável podendo diminuir o risco de várias doenças (diabetes, cardiovasculares, hipertensão, obesidade, acidente vascular cerebral, distúrbios gastrointestinais, melhora do perfil de lipídios séricos, função imunológica) (Hussain, et al., 2020). Sendo assim, um aspecto favorável ao consumo de bebidas vegetais relaciona-se com a presença de fibras em sua composição. Porém, analisando a tabela nutricional pode-se verificar que as bebidas vegetais aqui estudadas obtiveram valor médio de $0,4 \mathrm{~g}$ de fibras por $100 \mathrm{ml}$ de produto, o que representa apenas $1,6 \%$ da recomendação diária.

Comparando a tabela nutricional das bebidas vegetais e dos leites UHT (Tabela 3) infere-se que a concentração média 
do valor energético, teor de carboidratos, proteínas, gordura saturada e sódio foram maiores nos leites UHT. Em contrapartida, o teor médio de gordura total, fibras e cálcio os maiores resultados encontram-se nas bebidas vegetais. Um destaque pode ser dado ao teor de proteínas que se encontrou cerca de 3,4 vezes mais elevado nos leites UHT (valores médios de 3,1 g por 100 $\mathrm{ml}$ de produto) do que nas bebidas vegetais (valores médios de $0,9 \mathrm{~g}$ por $100 \mathrm{ml}$ ).

Sabe-se que o leite de vaca é considerado uma fonte importante de proteínas com aminoácidos essenciais e peptídeos bioativos de alta biodisponibilidade necessários para crescimento, reparo e manutenção da homeostase corporal e que se retirado ou substituído da alimentação pode resultar em deficiências nutricionais e desenvolvimento de doenças (Sousa \& Kopf, 2017). Além disso, outro destaque pode ser dado ao coeficiente de variação do teor de proteína para os leites UHT que foi de $6 \%$, diferentemente das bebidas vegetais que apresentaram coeficiente de variação de $107 \%$, sendo que este alto coeficiente pode ser correlacionado às mudanças nas fontes vegetais usadas nessas bebidas.

A Figura 1 representa a análise de componentes principais (PCA) estabelecida para avaliar as semelhanças e diferenças entre os atributos analisados na tabela nutricional das bebidas vegetais e leites UHT. É possível verificar que as amostras foram agrupadas, de acordo com suas semelhanças e diferenças, em quatro diferentes grupos.

As amostras encontradas na região amarela (Figura 1) são em sua totalidade leites UHT, exceto as amostras E1 e E3 que também ficaram dentro desta região, porém, são bebidas vegetais. Isso demonstra que, os leites, apesar da diferença do seu tipo (desnatado, semidesnatado e integral) apresentam atributos nutricionais (valor energético, teor de carboidratos, proteínas, gorduras, gorduras saturadas, fibras, sódio, cálcio) semelhantes entre si o que fazem eles permanecerem agrupados em uma dada região. As amostras de bebidas vegetais (E1 e E3) que se agruparam com os leites UHT são formuladas a base de soja, podendo assim, inferir, que dentre as bebidas vegetais analisadas (diferentes fontes vegetais), tais bebidas à base de soja foram aquelas que exibiram atributos nutricionais mais próximo do leite de origem animal. A soja é uma leguminosa de origem oriental asiática com uso difundido em todo o mundo por apresentar alto valor nutricional, sendo considerada uma boa fonte de proteínas e lipídeos, sendo utilizada na elaboração de proteínas vegetais texturizadas, concentrados e isolados de soja, suplementos proteicos, fórmulas infantis e bebidas vegetais (Verduc, et al., 2020). 
Tabela 1: Código da amostra, denominação e preço de venda, tempo de validade total em dias e tempo de consumo após aberto em dias das bebidas vegetais e dos leites UHT.

\begin{tabular}{|c|c|c|c|c|}
\hline Código da amostra & Denominação de venda & Preço de venda $(\mathrm{R} \$)$ & Tempo de validade total (dias) & Tempo de consumo após aberto (dias) \\
\hline A1 & Alimento com amêndoa & 6,99 & $*$ & Geladeira até 3 dias \\
\hline $\mathrm{A} 2$ & Alimento com amêndoa & 15,99 & 360 & Geladeira até 7 dias \\
\hline A3 & Alimento com amêndoa & 14,99 & $*$ & $*$ \\
\hline $\mathrm{B} 1$ & Alimento de arroz sabor original & 8,55 & 424 & Geladeira até 6 dias \\
\hline B2 & Alimento com arroz & 15,00 & $*$ & Geladeira até 2 dias \\
\hline B3 & Alimento com arroz & 16,79 & $*$ & Após o uso conservar refrigerado \\
\hline $\mathrm{C} 1$ & Alimento com castanha de caju orgânico & 26,56 & 365 & Geladeira até 7 dias \\
\hline $\mathrm{C} 2$ & Alimento com castanha de caju & 14,79 & 240 & Geladeira até 7 dias \\
\hline $\mathrm{C} 3$ & Alimento com castanha-de-caju & 16,79 & $*$ & Após aberto conservar refrigerado \\
\hline D1 & Alimento à base de leite de coco & 18,90 & $*$ & Geladeira até 5 dias \\
\hline D2 & Alimento com coco & 13,99 & 300 & Geladeira até 7 dias \\
\hline D3 & Alimento à base de leite de coco & 15,24 & $*$ & Geladeira até 3 dias \\
\hline E1 & Alimento com soja & 6,99 & $*$ & $*$ \\
\hline E2 & Alimento com soja & 14,33 & $*$ & Geladeira até 3 dias \\
\hline E3 & Alimento com soja & 8,90 & 180 & Geladeira até 3 dias \\
\hline $\mathrm{I} 1$ & Leite UAT (UHT) Integral & 4,19 & 120 & Geladeira até 2 dias \\
\hline $\mathrm{I} 2$ & Leite UHT Integral & 3,49 & 122 & Geladeira até 2 dias \\
\hline $\mathrm{I} 3$ & Leite UHT Integral & 3,59 & 153 & Geladeira até 2 dias \\
\hline I4 & Leite UHT Integral & 4,59 & 150 & Geladeira até 2 dias \\
\hline I5 & Leite UHT Integral & 3,99 & 153 & Geladeira até 2 dias \\
\hline SD1 & Leite UAT (UHT) Semidesnatado & 4,19 & 120 & Geladeira até 2 dias \\
\hline $\mathrm{SD} 2$ & Leite UHT Semidesnatado & 2,99 & 122 & Geladeira até 2 dias \\
\hline SD3 & Leite UHT Semidesnatado & 3,59 & 122 & Geladeira até 2 dias \\
\hline SD4 & Leite UHT Semidesnatado & 4,29 & 120 & Geladeira até 2 dias \\
\hline SD5 & Leite UHT Semidesnatado & 6,79 & 151 & Geladeira até 2 dias \\
\hline LD1 & Leite UAT (UHT) Desnatado & 4,19 & 120 & Geladeira até 2 dias \\
\hline LD2 & Leite UHT Desnatado & 3,29 & 122 & Geladeira até 2 dias \\
\hline LD3 & Leite UHT Desnatado & 3,59 & 122 & Geladeira até 2 dias \\
\hline LD4 & Leite UHT Desnatado & 4,59 & 120 & Geladeira até 2 dias \\
\hline LD5 & Leite UHT Desnatado & 5,29 & 153 & Geladeira até 2 dias \\
\hline
\end{tabular}

O símbolo $(*)$ refere-se às informações não apresentadas pelos fabricantes. Fonte: Autores. 
Research, Society and Development, v. 10, n. 13, e128101320860, 2021

(CC BY 4.0) | ISSN 2525-3409 | DOI: http://dx.doi.org/10.33448/rsd-v10i13.20860

Tabela 2: Tabela nutricional encontrada no rótulo das diferentes bebidas vegetais (padronizada para porção de $100 \mathrm{ml}$ ).

\begin{tabular}{|c|c|c|c|c|c|c|c|c|}
\hline \multirow[b]{2}{*}{ Bebida vegetal } & \multirow[b]{2}{*}{ Fonte vegetal } & \multicolumn{7}{|c|}{ INFORMAÇÃO NUTRICIONAL - Porção de $100 \mathrm{ml}$ (1/2 copo) } \\
\hline & & $\begin{array}{c}\text { Valor } \\
\text { Energético (kcal) }\end{array}$ & $\begin{array}{l}\text { Carboidratos } \\
(\mathrm{g})\end{array}$ & $\begin{array}{l}\text { Proteínas } \\
(\mathrm{g})\end{array}$ & $\begin{array}{l}\text { Gorduras } \\
(\mathrm{g})\end{array}$ & $\begin{array}{l}\text { Fibras } \\
(\mathrm{g})\end{array}$ & $\begin{array}{l}\text { Sódio } \\
(\mathrm{mg})\end{array}$ & $\begin{array}{l}\text { Cálcio } \\
(\mathrm{mg})\end{array}$ \\
\hline A1 & Amêndoa & 23,0 & 0,5 & 1,0 & 1,9 & 0,0 & 5,5 & 150,0 \\
\hline $\mathrm{A} 2$ & Amêndoa & 24,0 & 3,4 & 0,4 & 1,0 & 0,0 & 48,5 & 198,5 \\
\hline A3 & Amêndoa & 28,0 & 3,9 & 0,5 & 1,1 & 0,0 & 43,5 & 198,0 \\
\hline $\mathrm{B} 1$ & Arroz & 37,0 & 7,5 & 0,0 & 0,8 & 0,4 & 16,0 & 60,0 \\
\hline $\mathrm{B} 2$ & Arroz & 36,0 & 5,5 & 0,6 & 1,2 & 3,1 & 29,0 & 174,0 \\
\hline B3 & Arroz & 35,5 & 5,5 & 0,6 & 1,4 & 0,4 & 36,0 & 149,5 \\
\hline $\mathrm{C} 1$ & Castanha de Caju & 45,0 & 1,5 & 1,5 & 3,7 & 0,0 & 0,0 & 3,8 \\
\hline $\mathrm{C} 2$ & Castanha de Caju & 18,0 & 1,0 & 0,5 & 1,4 & 0,0 & 48,0 & 198,0 \\
\hline $\mathrm{C} 3$ & Castanha de Caju & 13,0 & 0,5 & 0,4 & 1,0 & 0,3 & 44,0 & 149,5 \\
\hline D1 & Coco & 33,5 & 0,5 & 0,4 & 3,3 & 0,8 & 5,5 & 125,0 \\
\hline D2 & Coco & 14,5 & 0,0 & 0,0 & 1,6 & 0,0 & 39,5 & 198,0 \\
\hline D3 & Coco & 31,5 & 1,5 & 0,3 & 2,7 & 0,4 & 7,5 & 0,0 \\
\hline E1 & Soja & 39,0 & 2,4 & 3,2 & 1,8 & 0,5 & 89,0 & 132,0 \\
\hline $\mathrm{E} 2$ & Soja & 34,0 & 2,6 & 2,2 & 1,6 & 0,0 & 35,5 & 137,5 \\
\hline E3 & Soja & 47,5 & 6,0 & 2,7 & 1,5 & 0,4 & 47,5 & 120,0 \\
\hline Média Geral & - & 30,6 & 2,8 & 0,9 & 1,7 & 0,4 & 33,0 & 132,9 \\
\hline Valor Máximo & - & 47,5 & 7,5 & 3,2 & 3,7 & 3,1 & 89,0 & 198,5 \\
\hline Valor Mínimo & - & 13,0 & 0,0 & 0,0 & 0,8 & 0,0 & 0,0 & 0,0 \\
\hline Amplitude & - & 34,5 & 7,5 & 3,2 & 2,9 & 3,1 & 89,0 & 198,5 \\
\hline Desvio padrão & - & 10,4 & 2,4 & 1,0 & 0,8 & 0,8 & 23,4 & 65,2 \\
\hline Erro padrão da média & - & 2,7 & 0,6 & 0,3 & 0,2 & 0,2 & 6,0 & 16,8 \\
\hline Coeficiente de variação & - & $34 \%$ & $85 \%$ & $107 \%$ & $49 \%$ & $186 \%$ & $71 \%$ & $49 \%$ \\
\hline
\end{tabular}

Fonte: Autores. 
Research, Society and Development, v. 10, n. 13, e128101320860, 2021

(CC BY 4.0) | ISSN 2525-3409 | DOI: http://dx.doi.org/10.33448/rsd-v10i13.20860

Tabela 3: Tabela nutricional encontrada nas diferentes marcas e tipos de leites UHT (padronizada para porção de $100 \mathrm{ml}$ ).

\begin{tabular}{|c|c|c|c|c|c|c|c|c|c|}
\hline \multirow[b]{2}{*}{ Leite UHT } & \multirow[b]{2}{*}{ Tipo } & \multicolumn{8}{|c|}{ INFORMAÇÃO NUTRICIONAL - Porção de $100 \mathrm{ml}$ (1/2 copo) } \\
\hline & & $\begin{array}{c}\text { Valor } \\
\text { Energético (kcal) }\end{array}$ & $\begin{array}{l}\text { Carboidratos } \\
\text { (g) }\end{array}$ & $\begin{array}{l}\text { Proteínas } \\
\text { (g) }\end{array}$ & $\begin{array}{l}\text { Gorduras } \\
(\mathrm{g})\end{array}$ & $\begin{array}{c}\text { Gorduras } \\
\text { saturadas (g) }\end{array}$ & $\begin{array}{l}\text { Fibras } \\
(\mathrm{g})\end{array}$ & $\begin{array}{c}\text { Sódio } \\
(\mathrm{mg})\end{array}$ & $\begin{array}{l}\text { Cálcio } \\
(\mathrm{mg})\end{array}$ \\
\hline I1 & Integral & 56,5 & 4,4 & 3,0 & 3,0 & 2,0 & 0,0 & 69,0 & 115,0 \\
\hline $\mathrm{I} 2$ & Integral & 58,5 & 5,0 & 2,9 & 3,0 & 2,0 & 0,0 & 52,5 & 120,0 \\
\hline $\mathrm{I} 3$ & Integral & 59,0 & 4,7 & 3,3 & 3,0 & 1,8 & 0,0 & 59,0 & 119,0 \\
\hline I4 & Integral & 59,0 & 5,0 & 3,0 & 3,0 & 2,0 & 0,0 & 99,0 & 126,0 \\
\hline I5 & Integral & 57,5 & 4,6 & 3,1 & 3,0 & 1,8 & 0,0 & 61,0 & 120,0 \\
\hline SD1 & Semi desnatado & 40,5 & 4,6 & 3,3 & 1,0 & 0,7 & 0,0 & 60,0 & 116,5 \\
\hline SD2 & Semi desnatado & 40,5 & 5,0 & 2,9 & 1,0 & 0,7 & 0,0 & 57,5 & 125,0 \\
\hline SD3 & Semi desnatado & 42,0 & 4,7 & 3,3 & 1,1 & 0,7 & 0,0 & 51,0 & 117,0 \\
\hline SD4 & Semi desnatado & 42,0 & 4,7 & 3,3 & 1,1 & 1,0 & 0,0 & 97,5 & 105,0 \\
\hline SD5 & Semi desnatado & 41,5 & 4,6 & 3,1 & 1,2 & 0,7 & 0,0 & 60,0 & 121,5 \\
\hline LD1 & Desnatado & 33,0 & 4,8 & 3,4 & 0,0 & 0,0 & 0,0 & 61,5 & 127,5 \\
\hline LD2 & Desnatado & 36,0 & 5,0 & 2,9 & 0,5 & 0,0 & 0,0 & 62,5 & 130,0 \\
\hline LD3 & Desnatado & 33,5 & 4,9 & 3,4 & 0,0 & 0,0 & 0,0 & 63,5 & 149,0 \\
\hline LD4 & Desnatado & 35,0 & 5,0 & 3,0 & 0,4 & 0,3 & 0,0 & 97,5 & 120,0 \\
\hline LD5 & Desnatado & 34,0 & 4,6 & 3,1 & 0,4 & 0,3 & 0,0 & 62,0 & 121,5 \\
\hline Média Geral & - & 44,6 & 4,8 & 3,1 & 1,4 & 0,9 & 0,0 & 67,6 & 122,2 \\
\hline Valor Máximo & - & 59,0 & 5,0 & 3,4 & 3,0 & 2,0 & 0,0 & 99,0 & 149,0 \\
\hline Valor Mínimo & - & 33,0 & 4,4 & 2,9 & 0,0 & 0,0 & 0,0 & 51,0 & 105,0 \\
\hline Amplitude & - & 26,0 & 0,6 & 0,5 & 3,0 & 2,0 & 0,0 & 48,0 & 44,0 \\
\hline Desvio padrão & - & 10,4 & 0,2 & 0,2 & 1,2 & 0,8 & 0,0 & 16,3 & 9,5 \\
\hline Erro padrão da média & - & 2,7 & 0,1 & 0,0 & 0,3 & 0,2 & 0,0 & 4,2 & 2,5 \\
\hline Coeficiente de variação & - & $23 \%$ & $4 \%$ & $6 \%$ & $84 \%$ & $86 \%$ & $0 \%$ & $24 \%$ & $8 \%$ \\
\hline
\end{tabular}

Fonte: Autores. 
Outros três agrupamentos puderam ser observados no PCA em questão, sendo todos eles caracterizados pela presença de bebidas vegetais. Analisando estes três grupos, verifica-se que a maior parte das bebidas vegetais permaneceram na região azul, mostrando que apesar das diferentes fontes vegetais as amostras exibem alguma semelhança nas características composicionais e nutricionais.

Figura 1: Gráfico de scores PC1 versus PC2 dos atributos encontrados nas tabelas nutricionais das bebidas vegetais e dos leites UHT (valor energético, teor de carboidratos, proteínas, gorduras, gorduras saturadas, fibras, sódio, cálcio).

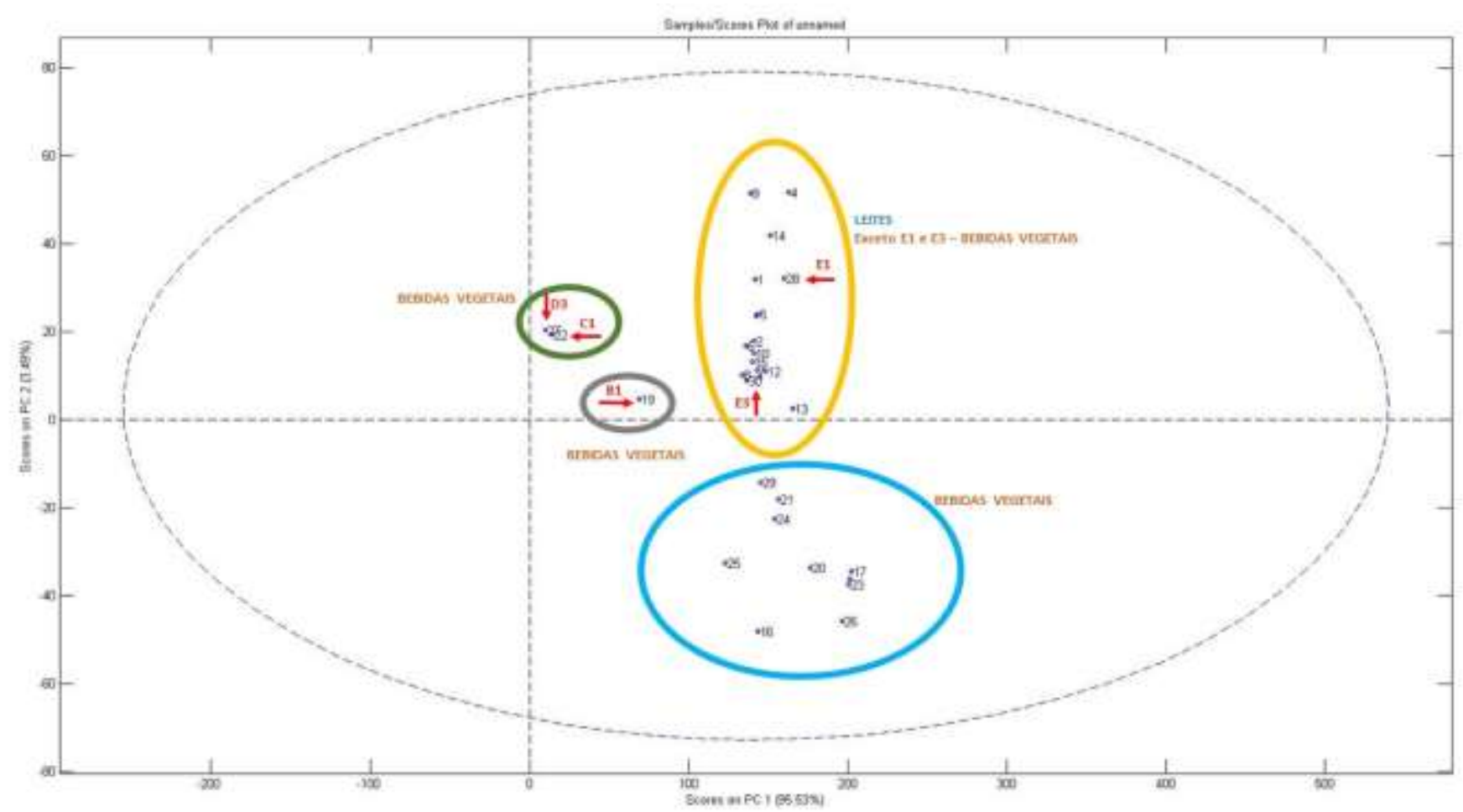

Fonte: Autores.

A Tabela 4 apresenta as listas de ingredientes presentes nos rótulos das bebidas vegetais. Pode-se verificar que as bebidas vegetais possuem uma extensa lista de ingredientes (água, açúcares, minerais, vitaminas, sal, estabilizantes, óleos dentre outros). Esta situação pode implicar em um menor consumo do produto, visto que é crescente o número de indivíduos que buscam consumir produtos "clean label” e/ou isentos de açúcares, conservantes ou outros aditivos alimentares (Venâncio, 2020).

A bebida vegetal da marca C1, castanha de caju orgânica (a qual apresentou o valor mais elevado de gordura dentre as amostras), possui a menor lista de ingredientes, contendo apenas água e castanha de caju orgânica, o que atende melhor a demanda dos indivíduos que buscam por produtos do tipo “clean label”. Em associação, destaca-se que as bebidas vegetais exibem adição de muitos aditivos como estabilizantes, emulsificantes e aromatizantes, sendo estes últimos utilizados principalmente com a finalidade de ocultar o sabor residual da bebida e proporcionar uma característica sensorial mais agradável ao paladar do consumidor (Sethi, et al., 2016). 
A Tabela 5 expõe as listas de ingredientes presentes nos rótulos dos leites UHT. É possível verificar que os leites exibem majoritariamente nas listas de ingredientes leite e estabilizantes como: citrato de sódio, trifosfato de sódio, difosfato de sódio e monofosfato de sódio. Estes compostos (estabilizantes) já se encontram presentes na composição do leite natural (porém em concentrações variáveis, considerando a biossíntese de cada animal), e quando adicionados ao mesmo, garantem a estabilidade das proteínas durante o processo UHT e o armazenamento do produto (Boumpa, et al., 2008). De acordo com Regulamento Técnico de Identidade e Qualidade do Leite UHT é aceito o uso dos seguintes estabilizantes: citrato de sódio, monofosfato de sódio, difosfato de sódio e trifosfato de sódio, separados ou em combinação, em uma quantidade não superior a $0,1 \mathrm{~g} / 100 \mathrm{ml}$ expressos em $\mathrm{P}_{2} 0_{5}$ (Brasil, 1997), ou seja, é permitida apenas a adição de 4 diferentes aditivos ao leite UHT, sendo proibida a adição de outros como conservantes, aromatizantes, antioxidantes ou emulsificantes.

As alegações apresentadas na parte frontal do cada rótulo das bebidas vegetais e dos leites UHT estão apresentadas nas Tabelas 6 e 7, respectivamente. Tais atributos são caracterizados como informações nutricionais complementares e sugerem que um alimento possui propriedades nutricionais particulares. Segundo a RDC nº 54/2012 (Brasil, 2012) é obrigatório declarar na tabela nutricional a quantidade de qualquer nutriente que se faça algum tipo de alegação, inclusive quando se declara que "não contém". Vale ressaltar que as alegações referentes às características inerentes dos alimentos devam apresentar no rótulo um esclarecimento de que todos os alimentos desse tipo também possuem essas mesmas características. Além disso, as declarações de presença ou ausência de glúten e lactose devem seguir legislações específicas (Brasil, 2017a; Brasil, 2017b; Brasil, 2003).

De acordo com a Tabela 6, verifica-se que as bebidas à base de vegetais apresentaram um maior número de alegações em seus rótulos do que os leites UHT (Tabela 7), tendo sido encontrado uma média de 4,5 alegações por rótulos de bebidas vegetais e 1 alegação por rótulo de leite UHT. Destaca-se que as bebidas vegetais B3 e C3 apresentaram um total de 9 alegações cada.

Estudos demonstram que os consumidores dão preferência à compra de produtos que apresentem informações nutricionais complementares em seus rótulos. Todavia, constatou-se que poucos desses consumidores são capazes de fazerem um julgamento crítico a cerca dessas alegações (Bauduín \& Romero, 2020; Morais, et al., 2020). 
Tabela 4: Lista de ingredientes exibidas no rótulo de diferentes marcas e fontes vegetais de bebidas vegetais.

\begin{tabular}{|c|c|c|}
\hline Bebida vegetal & Fonte Vegetal & Lista de ingredientes \\
\hline A1 & Amêndoa & $\begin{array}{l}\text { Água, amêndoa, maltodextrina, minerais fosfato de cálcio tribásico e sulfato de zinco, vitaminas E, B6, A, ácido fólico e B12, aromatizante, } \\
\text { estabilizantes: goma gelana e goma xantana e regulador de acidez citrato de sódio. }\end{array}$ \\
\hline $\mathrm{A} 2$ & Amêndoa & $\begin{array}{l}\text { Pasta de amêndoas reconstituída, açúcar, minerais (cálcio e zinco), sal marinho, vitaminas (B2, D2 e B12), estabilizantes gomas alfarroba e gelana } \\
\text { e emulsificante lecitina de girassol. }\end{array}$ \\
\hline A3 & Amêndoa & $\begin{array}{l}\text { Água, açúcar, amêndoa, maltodextrina, minerais (cálcio e zinco), sal, vitaminas (E, B6, A, ácido fólico, D e B12), aromatizante, emulsificante } \\
\text { lecitina de girassol e estabilizantes goma xantana e goma gelana. }\end{array}$ \\
\hline B1 & Arroz & $\begin{array}{l}\text { Água, arroz, óleo de girassol, carbonato de cálcio (fonte de cálcio), sal marinho, emulsificante natural goma xantana e estabilizantes fosfato } \\
\text { tricálcio e citrato de sódio. }\end{array}$ \\
\hline $\mathrm{B} 2$ & Arroz & $\begin{array}{l}\text { Água, arroz integral }(6,6 \%) \text {, fibra solúvel (polidextrose), óleo vegetal de girassol, cálcio (fosfato tricálcico), sal, vitamina D (colicalciferol), } \\
\text { estabilizante goma gelana, aromatizantes e emulsificante lecitina de soja. }\end{array}$ \\
\hline B3 & Arroz & $\begin{array}{l}\text { Água, arroz, óleo vegetal de girassol, cálcio (trifosfato tricálcico), sal marinho, vitamina A (palmitato de retinila), vitamina D2 (ergocalciferol), } \\
\text { estabilizante natural goma gelana e aromatizante natural. }\end{array}$ \\
\hline $\mathrm{C} 1$ & Castanha de Caju & Água e castanha de caju orgânica. \\
\hline $\mathrm{C} 2$ & Castanha de Caju & $\begin{array}{l}\text { Pasta de castanha de caju reconstituída, minerais (cálcio e zinco), sal marinho, vitaminas (B2, D2 e B12), estabilizantes gomas alfarroba e gelana; } \\
\text { emulsificante lecitina de girassol, aromas naturais e antioxidante ascorbato de sódio. }\end{array}$ \\
\hline C3 & Castanha de Caju & $\begin{array}{l}\text { Pasta de castanha-de-caju reconstituída, cálcio (fosfato tricálcico), sal marinho, vitamina A (palmitato de retinila), vitamina D2 (ergocalciferol), } \\
\text { estabilizante natural goma gelana e emulsificante natural lecitina de girassol. }\end{array}$ \\
\hline D1 & Coco & Água, leite de coco, fosfato tricálcico, vitaminas (A,D, B12), aromatizantes naturais, espessante goma alfarroba e estabilizante goma gelatina. \\
\hline D2 & Coco & $\begin{array}{l}\text { Leite de coco reconstituído, minerais (cálcio e zinco), sal marinho, vitaminas B2, D2 e B12), aromas naturais, emulsificante lecitina de girassol, } \\
\text { estabilizantes gomas alfarroba e geleana e antioxidante ascorbato de sódio. }\end{array}$ \\
\hline D3 & Coco & $\begin{array}{l}\text { Água, leite de coco, emulsificante celulose microcristalina, estabilizantes gomas xantana e carboximetilcelulose (CMC), acidulante ácido cítrucim } \\
\text { conservantes sorbato de potássio e benzoato de sódio, aromatizantes idênticos ao natural. }\end{array}$ \\
\hline E1 & Soja & $\begin{array}{l}\text { Água, grãos de soja, açúcar, minerais (cálcio e zinco), sal, vitaminas (E, B6, A, ácido fólico, D e B12), aromatizante, estabilizantes: citrato de } \\
\text { sódio, goma gelana e goma xantana, emulsificante lecitina de soja e edulcorante sucralose. }\end{array}$ \\
\hline E2 & Soja & $\begin{array}{l}\text { Extrato de soja, água, açúcar, carbonato de cálcio, sal, vitaminas A e D, aromatizante, emulsificante: lecitina de soja, estabilizante: goma } \\
\text { carragena, antiespumante: polidimetilsiloxano e edulcorante: sucralose. }\end{array}$ \\
\hline E3 & Soja & $\begin{array}{l}\text { Água, extrato de soja, açúcar, cálcio (fosfato tricálcico), cloreto de sódio (sal), estabilizantes carragena e carboximetilcelulose, aromatizantes e } \\
\text { estabilizante citrato de sódio. }\end{array}$ \\
\hline
\end{tabular}


Tabela 5: Lista de ingredientes apresentada no rótulo de diferentes marcas e tipos de leites UHT.

\begin{tabular}{|c|c|c|}
\hline Leite UHT & Tipo & Lista de ingredientes \\
\hline I1 & Integral & Leite integral, estabilizante citrato de sódio e mistura de estabilizantes para leite UHT (trifosfato de sódio, difosfato de sódio e monofosfato de sódio). \\
\hline I2 & Integral & Leite integral e estabilizantes citrato de sódio, trifosfato de sódio, monofosfato monossódio e difosfato dissódico. \\
\hline I3 & Integral & Leite integral, vitaminas (C, A e D) e estabilizantes (citrato de sódio, trifosfato de sódio, difosfato de sódio e monofosfato de sódio). \\
\hline I4 & Integral & Leite integral, estabilizantes trifosfato de sódio, difosfato de sódio e monofosfato de sódio. \\
\hline I5 & Integral & Leite integral e estabilizantes citrato de sódio, trifosfato de sódio, monofosfato de sódio e difosfato de sódio. \\
\hline SD1 & Semi desnatado & Leite semidesnatado e estabilizantes citrato de sódio, trifosfato de sódio, dihidrogênio difosfato dissódico e monofosfato monossódico. \\
\hline SD2 & Semi desnatado & Leite semidesnatado e estabilizantes citrato de sódio, trifosfato de sódio, monofosfato monossódio e difosfato dissódico. \\
\hline SD3 & Semi desnatado & Leite semidestanatado, vitaminas (C, A e D), estabilizantes (citrato de sódio, trifosfato de sódio, difosfato de sódio e monofosfato de sódio). \\
\hline SD4 & Semi desnatado & Leite semidesnatado, estabilizantes trifosfato de sódio, difosfato de sódio e monofosfato de sódio. \\
\hline SD5 & Semi desnatado & Leite semidesnatado e estabilizantes trifosfato de sódio, citrato de sódio, monofosfato de sódio e difosfato de sódio. \\
\hline LD1 & Desnatado & Leite desnatado e estabilizantes citrato de sódio, trifosfato de sódio, dihidrogênio difosfato dissódico e monofosfato monossódico. \\
\hline LD2 & Desnatado & Leite desnatado e estabilizantes citrato de sódio, trifosfato de sódio, monofosfato monossódio e difosfato dissódico. \\
\hline LD3 & Desnatado & Leite desnatado, cálcio (fosfato tricálcico) e estabilizantes (citrato de sódio, trifosfato de sódio, difosfato de sódio e monofosfato de sódio). \\
\hline LD4 & Desnatado & Leite desnatado, estabilizantes trifosfato de sódio, difosfato de sódio e monofosfato de sódio. \\
\hline LD5 & Desnatado & Leite desnatado e estabilizantes trifosfato de sódio, citrato de sódio, monofosfato de sódio e difosfato de sódio. \\
\hline
\end{tabular}

Fonte: Autores. 
Research, Society and Development, v. 10, n. 13, e128101320860, 2021

(CC BY 4.0) | ISSN 2525-3409 | DOI: http://dx.doi.org/10.33448/rsd-v10i13.20860

Tabela 6: Lista de alegações ou atributos apresentados no display frontal dos rótulos de diferentes marcas e tipos de bebidas vegetais.

\begin{tabular}{|c|c|c|c|c|c|c|c|c|c|c|c|c|c|c|c|c|}
\hline \multirow{3}{*}{ Alegações ou atributos } & \multirow{3}{*}{$\begin{array}{l}\text { Número } \\
\text { de citações }\end{array}$} & \multicolumn{15}{|c|}{ Fontes vegetais das bebidas por marcas } \\
\hline & & \multicolumn{3}{|c|}{ Amêndoa } & \multicolumn{3}{|c|}{ Arroz } & \multicolumn{3}{|c|}{ Castanha de Caju } & \multicolumn{3}{|c|}{ Coco } & \multicolumn{3}{|c|}{ Soja } \\
\hline & & A1 & $\mathrm{A} 2$ & A3 & B1 & $\mathrm{B} 2$ & B3 & $\mathrm{C} 1$ & $\mathrm{C} 2$ & $\mathrm{C} 3$ & D1 & D2 & D3 & E1 & E2 & E3 \\
\hline Sem lactose ${ }^{* 1}$ & 10 & $\mathrm{x}$ & $\mathrm{x}$ & $\mathrm{x}$ & $\mathrm{x}$ & & $\mathrm{x}$ & & $\mathrm{x}$ & $\mathrm{x}$ & $\mathrm{x}$ & $\mathrm{x}$ & & $\mathrm{x}$ & & \\
\hline Sem adição de açúcares ou zero açúcar & 9 & $\mathrm{x}$ & & & $\mathrm{x}$ & & $\mathrm{x}$ & $\mathrm{x}$ & $\mathrm{x}$ & $\mathrm{x}$ & $\mathrm{x}$ & $\mathrm{x}$ & $\mathrm{x}$ & & & \\
\hline Sem soja & 5 & & & $\mathrm{x}$ & & & $\mathrm{x}$ & $\mathrm{x}$ & & $\mathrm{x}$ & & & $\mathrm{x}$ & & & \\
\hline Sem glúten*2 & 4 & & & & $\mathrm{x}$ & & $\mathrm{x}$ & $\mathrm{x}$ & & $\mathrm{x}$ & & & & & & \\
\hline Sem Colesterol / Zero Colesterol & 4 & $\mathrm{x}$ & & & $\mathrm{x}$ & & $\mathrm{x}$ & & & $\mathrm{x}$ & & & & & & \\
\hline Sem lácteos & 1 & & & & & & & $\mathrm{x}$ & & & & & & & & \\
\hline Sem conservantes & 1 & & & & & & & $\mathrm{x}$ & & & & & & & & \\
\hline Light em valor energético & 1 & & & & & & & & & & & & & & $\mathrm{x}$ & \\
\hline Rico em cálcio & 4 & $\mathrm{x}$ & & $\mathrm{x}$ & & & $\mathrm{x}$ & & & $\mathrm{x}$ & & & & & & \\
\hline Fonte de cálcio & 4 & & & & & & & & & & $\mathrm{x}$ & & & $\mathrm{x}$ & $\mathrm{x}$ & $\mathrm{x}$ \\
\hline $40 \%$ do cálcio & 3 & & $\mathrm{x}$ & & & & & & $\mathrm{x}$ & & & $\mathrm{x}$ & & & & \\
\hline Fonte de Vitamina A & 3 & $\mathrm{x}$ & & & & & $\mathrm{x}$ & & & $\mathrm{x}$ & & & & & & \\
\hline Fonte de Vitamina D & 2 & & & & & & $\mathrm{x}$ & & & $\mathrm{x}$ & & & & & & \\
\hline Fonte de Vitamina B6 & 1 & $\mathrm{x}$ & & & & & & & & & & & & & & \\
\hline Fonte de Vitamina B12 & 1 & $\mathrm{x}$ & & & & & & & & & & & & & & \\
\hline Fonte de Vitamina E & 1 & $\mathrm{x}$ & & & & & & & & & & & & & & \\
\hline Fonte de proteína & 1 & & & & & & & & & & & & & $\mathrm{x}$ & & \\
\hline $100 \%$ vegetal / Origem Vegetal & 7 & & $\mathrm{x}$ & & & $\mathrm{x}$ & $\mathrm{x}$ & & $\mathrm{x}$ & $\mathrm{x}$ & $\mathrm{x}$ & $\mathrm{x}$ & & & & \\
\hline $100 \%$ Vegano & 2 & & & & & & & & & & $\mathrm{x}$ & & $\mathrm{x}$ & & & \\
\hline $100 \%$ natural & 1 & & & & & & & $\mathrm{x}$ & & & & & & & & \\
\hline $100 \%$ cereal & 1 & & & & & $\mathrm{x}$ & & & & & & & & & & \\
\hline Leite de coco pronto para beber & 2 & & & & & & & & & & $\mathrm{x}$ & & $\mathrm{x}$ & & & \\
\hline Total de alegações ou atributos por prod & nível no merc & 8 & 3 & 3 & 4 & 2 & 9 & 6 & 4 & 9 & 6 & 4 & 4 & 3 & 2 & 1 \\
\hline
\end{tabular}

${ }^{* 1}$ Deve seguir a resolução RDC n ${ }^{\circ}$ 135/2017 quando da presença de lactose e RDC n 136/2017 quando da ausência de lactose nos alimentos e bebidas.

${ }^{* 2}$ Deve seguir a lei ${ }^{\circ}$ 10.674/2003 que determina que todos os alimentos e bebidas devam informar sobre a presença ou ausência do glúten.

Fonte: Autores. 
Research, Society and Development, v. 10, n. 13, e128101320860, 2021

(CC BY 4.0) | ISSN 2525-3409 | DOI: http://dx.doi.org/10.33448/rsd-v10i13.20860

Tabela 7: Lista de alegações ou atributos apresentados no display frontal dos rótulos de diferentes marcas e tipos de leites UHT.

\begin{tabular}{|c|c|c|c|c|c|c|c|c|c|c|c|c|c|c|c|c|}
\hline \multirow{3}{*}{ Alegações ou atributos } & \multirow{3}{*}{ Número de citações } & \multicolumn{15}{|c|}{ Tipos e marcas de leites UHT } \\
\hline & & \multicolumn{5}{|c|}{ Integral } & \multicolumn{5}{|c|}{ Semi desnatado } & \multicolumn{5}{|c|}{ Desnatado } \\
\hline & & I1 & $\mathrm{I} 2$ & I3 & I4 & I5 & SD1 & $\mathrm{SD} 2$ & SD3 & SD4 & SD5 & LD1 & LD2 & LD3 & LD4 & LD5 \\
\hline Fonte de cálcio & 3 & $X$ & & & & & $\mathrm{x}$ & & & & & $\mathrm{x}$ & & & & \\
\hline Rico em cálcio & 1 & & & & & & & & & & & & & $\mathrm{x}$ & & \\
\hline Rico em vitamina $\mathrm{A}$ & 2 & & & $\mathrm{x}$ & & & & & $\mathrm{X}$ & & & & & & & \\
\hline Rico em vitamina $\mathrm{C}$ & 2 & & & $\mathrm{x}$ & & & & & $X$ & & & & & & & \\
\hline Rico em vitamina D & 2 & & & $\mathrm{x}$ & & & & & $\mathrm{X}$ & & & & & & & \\
\hline Teor de gordura $3 \%$ & 3 & & $\mathrm{x}$ & & $\mathrm{x}$ & $\mathrm{x}$ & & & & & & & & & & \\
\hline Teor de gordura $1,1 \%$ & 1 & & & & & & & & & $\mathrm{x}$ & & & & & & \\
\hline Teor de gordura $1 \%$ & 1 & & & & & & & $\mathrm{x}$ & & & & & & & & \\
\hline 83 kcal/porção & 1 & & & & & & & & & & $\mathrm{x}$ & & & & & \\
\hline 68 kcal/porção & 1 & & & & & & & & & & & & & & & $\mathrm{x}$ \\
\hline \multicolumn{2}{|c|}{ Total de alegações ou atributos por produto disponível no mercado } & 1 & 1 & 3 & 1 & 1 & 1 & 1 & 3 & 1 & 1 & 1 & 0 & 1 & 0 & 1 \\
\hline
\end{tabular}

Fonte: Autores. 


\section{Conclusão}

Comparando as informações disponibilizadas nos rótulos das bebidas vegetais e dos leites UHT pode-se inferir que há uma maior padronização nos atributos nutricionais assim como na lista de ingredientes, tempo de validade total, claims e denominação de venda para os leites UHT. Esta situação é resultante da existência de uma legislação de identidade e qualidade do leite, já amplamente utilizada e consolidada, o que se diferencia da ausência de aspectos regulatórios específicos para bebidas vegetais até o presente momento. Destaca-se ainda que, comparando a tabela nutricional das bebidas vegetais e dos leites UHT, infere-se que o valor energético médio, e as concentrações médias de carboidratos, proteínas, gordura saturada e sódio foram maiores nos leites UHT. Em contrapartida, os teores médios de gordura total, fibras e cálcio tiveram os maiores resultados encontrados nas bebidas vegetais. Além disto, o preço de mercado do leite de vaca é bem inferior quanto se compara com as bebidas vegetais, sendo que o custo mais elevado destes produtos ocorre principalmente pelo tipo de processamento usado assim como pelos desafios de alto valor comercial agregado dos vegetais e a dificuldades de realizar fabricações em grande escala. Por fim, a análise de componentes principais (PCA) possibilitou agrupar as amostras de acordo com as similaridades dos seus atributos nutricionais em basicamente duas grandes regiões: leites UHT e bebidas vegetais. Destaca-se neste PCA, a maior similaridade dos atributos nutricionais das bebidas vegetais a base de soja com o leite UHT e a existência de valores discrepantes de alguns atributos analisados para 3 bebidas vegetais (arroz, castanha e coco).

\section{Agradecimentos}

À Universidade Federal de Juiz de Fora (Departamento de Química e Faculdade de Farmácia) e ao Conselho Nacional de Desenvolvimento Científico e Tecnológico (CNPq) pelas bolsas 307334/2020-1 e 315337/ 2018-4, assim como pelo projeto Doutorado Académico de Inovação (DAI).

\section{Referências}

Aydar, E. F., Tutuncu, S., \& Ozcelik, B. (2020). Plant-based milk substitutes: Bioactive compounds, conventional and novel processes, bioavailability studies, and health effects. Journal of Functional Foods, 70, 1-15. https://doi.org/10.1016/j.jff.2020.103975

Boumpa, T., Tsioulpas, A., Grandison, A. S., \& Lewis, M. J. (2008). Effects of phosphates and citrates on sediment formation in UHT goats' milk. Journal of Dairy Research, 75(2), 160-166. https://doi.org/10.1017/S0022029908003166

Baudín, F. A., \& Romero, M. C. (2020). Comprensión de los consumidores del etiquetado nutricional para la compra de alimentos envasados. Revista española de nutrición comunitaria. Spanish journal of community nutrition, 26(3), 1-14.

Brasil. Lei no 8.918, de 14 de julho de 1994. Ministério da agricultura, do abastecimento e da reforma agrária; Ministério da Saúde - MS. Diário Oficial da União: Brasília, DF, 14 jul. 1994.

Brasil. Ministério da Agricultura, do Abastecimento e da Reforma Agrária. Portaria no 370, de 4 de setembro de 1997. Aprova o Regulamento Técnico de Identidade e Qualidade do Leite UAT (UHT). Diário Oficial da República Federativa do Brasil, Brasília, 8 set. 1997. Seção 1, p. 19700.

Brasil. Agência Nacional de Vigilância Sanitária. Resolução RDC n. 54, de 12 de novembro de 2012. Dispõe sobre o Regulamento Técnico sobre Informação Nutricional Complementar. 2012. Diário Oficial da República Federativa do Brasil, Brasília, nov. 2012. Seção 1, p.3.

Brasil. Resolução da Diretoria Colegiada $\mathrm{n}^{\circ}$ 259, de 20 de setembro de 2002. Regulamento Técnico de Rotulagem de Alimentos Embalados. ANVISA Agência Nacional de Vigilância Sanitária.

Brasil. Lei n.10.674, 16 de maio de 2003. O Congresso Nacional obriga que os produtos alimentícios comercializados informem sobre a presença de glúten. Diário Oficial da União. 200319 maio; (94):1; Seção 1.

Brasil. Ministério da Saúde. Agência Nacional de Vigilância Sanitária (ANVISA). Resolução RDC no 135, de 8 de Fevereiro de $2017 a$. Aprova o regulamento técnico referente a alimentos para fins especiais, para dispor sobre os alimentos para dietas com restrição de lactose. Diretoria Colegiada da Agência Nacional de Vigilância Sanitária, Brasília, 31 de Janeiro de 2017.

Brasil. Agência nacional de vigilância sanitária. $R D C n^{o} 136$, de 9 de fevereiro de $2017 b$. Estabelece os requisitos para declaração obrigatória da presença de lactose nos rótulos dos alimentos.

Cardoso, C. F., Cruz, A. G., Pinto, U. M., \& Faria, J. A. F. (2012). Investigating the adulteration of UHT milk in Brazil. In Case Studies in Food Safety and Authenticity (pp. 301-307). Woodhead Publishing. 
Research, Society and Development, v. 10, n. 13, e128101320860, 2021

(CC BY 4.0) | ISSN 2525-3409 | DOI: http://dx.doi.org/10.33448/rsd-v10i13.20860

Casé, F., Deliza, R., \& Rosenthal, A. (2005). Produção de'leite'de soja enriquecido com cálcio. Food Science and Technology, 25, 86-91. https://doi.org/10.1590/S0101-20612005000100014

Cirilo, G. M. D., Olivieri, C. M. R., \& Martins, M. C. T. (2020). Bebidas vegetais alternativas ao leite. Life Style, 7(1), 15-25. https://doi.org/10.19141/22373756.lifestyle.v7.n1.p15-25

Hussain, S., Jõudu, I., \& Bhat, R. (2020). Dietary fiber from underutilized plant resources-a positive approach for valorization of fruit and vegetable wastes. Sustainability, 12(13), 1-30. https://doi.org/10.3390/su12135401

Ludke, M. \& Andre, M. E. D. A. (2013). Pesquisas em educação: uma abordagem qualitativa. São Paulo: E.P.U.

Matlab. (2010). (Versão 7.10.0) [Software]. United States, USA: MathWorks Inc.

McClements, D. J., Newman, E., \& McClements, I. F. (2019). Plant-based milks: A review of the science underpinning their design, fabrication, and performance. Comprehensive reviews in food science and food safety, 18(6), 2047-2067. https://doi.org/10.1111/1541-4337.12505

MILKPOINT. Mercado de bebidas vegetais cresce dois dígitos no Brasil. 2018. 〈https://bit.ly/2PxqeKo>. Acesso em: 21 jul. 2021.

Morais, A. C. B., Fiori, S. L., Bertin, R. L., \& Medeiros, C. O. (2020). Conhecimento e uso de rótulos nutricionais por consumidores. DEMETRA: Alimentação, Nutrição \& Saúde, 15, 1-15. https://doi.org/10.12957/demetra.2020.45847

Munekata, P. E., Domínguez, R., Budaraju, S., Soto, R. E., Barba, F. J., Mallikarjunan, K., Roohinejad, S., \& Lorenzo, J. M. (2020). Effect of innovative food processing technologies on the physicochemical and nutritional properties and quality of non-dairy plant-based beverages. Foods, 9(3), 1-16. https://doi.org/10.3390/foods9030288

Paul, A. A., Kumar, S., Kumar, V., \& Sharma, R. (2020). Milk Analog: Plant based alternatives to conventional milk, production, potential and health concerns. Critical reviews in food science and nutrition, 60(18), 3005-3023. https://doi.org/10.1080/10408398.2019.1674243

Sethi, S., Tyagi, S. K., \& Anurag, R. K. (2016). Plant-based milk alternatives an emerging segment of functional beverages: a review. Journal of food science and technology, 53(9), 3408-3423. https://doi.org/10.1007/s13197-016-2328-3

R Foundation for Statistical Computing Platform. (2020). (Versão 4.1.1) [Software].

Sousa, A., \& Kopf, K. A. (2017). Nutritional Implications of an Increasing Consumption of Non-Dairy Plant-Based Beverages Instead of Cow's Milk in Switzerland. Advances in Dairy Research, 5(04), 1-7. https://doi.org/10.4172/2329-888X.1000197

Venâncio, D. P., \& Pandolfi, M. A. C. (2020). Clean label na comercialização de produtos. Revista Interface Tecnológica, 17(2), 535-541. https://doi.org/10.31510/infa.v17i2.907

Verduci, E., Di Profio, E., Cerrato, L., Nuzzi, G., Riva, L., Vizzari, G., D'Auria, E., Giannì, M. L., Zuccotti, G., \& Peroni, D. G. (2020). Use of soy-based formulas and cow's milk allergy: lights and shadows. Frontiers in Pediatrics, 8, 1-11. https://doi.org/10.3389/fped.2020.591988 